\title{
Inelastic Deformation and Damage at High Temperature
}

\author{
Grant DE-FG02-86ER13566
}

\author{
Erhard Krempl \\ Mechanics of Materials Laboratory \\ Department of Mechanical Engineering, \\ Aeronautical Engineering \& Mechanics \\ Rensselaer Polytechnic Institute \\ Troy, N.Y. 12180-3590
}

Progress Report April 1, 1992 through April 30, 1993

\section{Summary}

Combined experimental and theoretical investigations into the inelastic deformation and damage behavior of engineering alloys at elevated temperatures are being pursued. Modeling of effects of recovery of state observed in modified $9 \mathrm{Cr}-1 \mathrm{Mo}$ steel has been completed and two papers on this subject have been accepted by Materials Science and Engineering. Finite deformation formulations of the viscoplasticity theory based on overstress (VBO) include a modified growth law for the equilibrium stress and a rationale for choosing objective derivatives of stress-like state variables. Numerical simulations are in progress and a paper will be presented at the forthcoming Fourth International Symposium on Plasticity and Its Current Applications. Seven biaxial low-cycle fatigue tests at $538^{\circ} \mathrm{C}$ have been completed with the reversing DC potential drop apparatus attached. A new method of data analysis and smoothing was developed which showed a significant increase in voltage drop in the area of crack formation. Correlation with solutions of Laplace's Equation for a semi-elliptical crack showed similar shapes for the voltage drop. A paper was presented at the Eleventh Symposium on Energy Engineering Sciences.

\section{Personnel}

The principal investigator is Dr. Erhard Krempl, Rosalind and John J. Redfern Jr. Professor of Engineering ( $10 \%$ of academic year time), assisted by Brian P. Fitzgerald (BS, Physics, RPI, 1981), PhD candidate (full time); Patrick S. Majors, PhD candidate (full time); and Instrumentation Engineer William Mielke (20\% time). Brian is in his tifth year on the program and is responsible for the experimental investigation using the reversing DC potential drop method as a potential measure of damage and crack growth. Finite deformation and modeling of recovery in the context of the viscoplasticity theory based on overstress are the subjects of the theoretical studies of Pat who is in the third year on the program.

\section{DISCLAIMER}

This report was prepared as an account of work sponsored by an agency of the United States Government. Neither the United States Government nor any agency thereof, nor any of their employees, makes any warranty, express or implied, or assumes any legal liability or responsibility for the accuracy, completeness, or usefulness of any information, apparatus, product, or process disclosed, or represents that its use would not infringe privately owned rights. Reference herein to any specific commercial product, process, or service by trade name, trademark, manufacturer, or otherwise does not necessarily constitute or imply its endorsement, recommendation, or favoring by the United States Government or any agency thereof. The views and opinions of authors expressed herein do not lecessarily state or reflect those of the

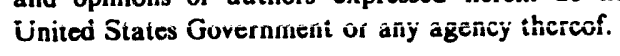

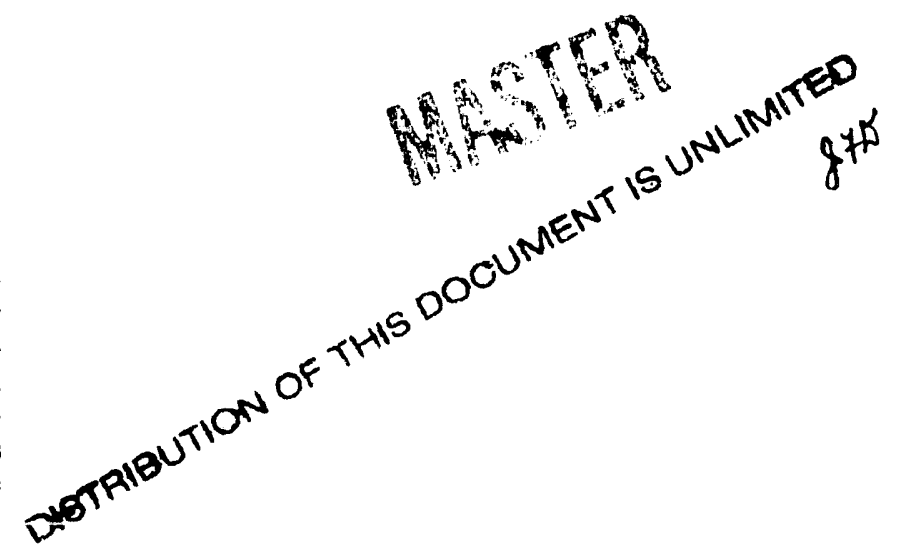




\section{Technical Progress}

\section{Publications}

"Viscoplasticity Theory Based on Overstress: The Modeling of Ratcheting and Cyclic Hardening of AISI Type 304 Stainless Steel," E. Krempl and S. H. Choi, Nuclear Engineering and Design, 193, 401-410 (1992).

"Viscoplasticity Theory Based on Overstress. Comparison of Two Growth Laws for the Equilibrium Stress," E. Krempl and J. M. Gleason, Proceedings of the 1992 Annual Meeting of JSME/MMD, Hokkaido University, Sapporo, Japan, The Japan Society of Mechanical Engineers, Tokyo, Japan, 87-89 (1992).

"The Rate Dependent Behavior of Modified 9Cr-1MO Steel. Some Experimental Results at $538^{\circ}$ C," M. B. Ruggles, S. Cheng and E. Krempl, to appear in Materials Science and Engineering, A, 1993.

"The Rate Dependent Behavior of Modified 9Cr-1Mo Steel. Modeling Using the Isotropic Viscoplasticity Theory Based on Overstress," P. S. Majors and E. Krempl, to appear in Materials Science and Engineering, A, 1993.

"Viscoplasticity Theory Based on Overstress: The Modeling of Biaxial Cyclic Hardening Using Irreversible Plastic Strain," S. H. Choi and E. Krempl, to appear in ASTM STP $1191,1993$.

"An Incremental Life Prediction Law for Multiaxial Creep-Fatigue Interaction and Thermomechanical Loading," N. M. Yeh and E. Krempl, to appear in ASTM STP 1191, 1993.

\section{Presentations $-\underline{\text { Invited }}$}

"Viscoplasticity Theory Based on Overstress. Comparison of Two Growth Laws for the Equilibrium Stress," E. Krempl and J. M. Gleason, 1992 Annual Meeting of JSME/MMD, Hokkaido University, Sapporo, Japan, September 2-3, 1992.

"Potential Drop Crack Growth Monitoring in High Temperature Biaxial Fatigue Tests," B. P. Fitzgerald and E. Krempl, Eleventh Symposium on Energy Engineering Sciences, Argonne National Laboratory, Argonne, IL, May 3-5, 1993.

\section{Short Technical Description}

\section{Modeling and Theoretical Studies}

New experimental evidence about cycle dependent softening of modified $9 \mathrm{Cr}-1 \mathrm{Mo}$ steel at room temperature led to a reinterpretation of static recovery within the previously developed Viscoplasticity Theory Based on Overstress (VBO) with recovery of state. The data indicate that much of the observed softening, which we previously attributed to recovery of state, is also present at room temperature, and therefore cannot be classified as recovery of state in the commonly accepted sense. These developments are explained in detail in the papers by Ruggles, Cheng and Krempl and Majors and Krempl which are in print in Materials Science and Engineering, A. 
The further development of the finite deformation version of VBO has been guided by the observation from scarce experimental data that different materials can exhibit very different qualitative behavior at large deformations. As such, it has been our goal to develop guidelines and a kinematic basis which will allow a user to specialize the basic constitutive structure of VBO to a variety of materials and situations. The framework being used allows us to propose, for example, various objective growth laws for the kinematic stress, a variable which can be used to model induced anisotropy and second order effects. Furthermore, an improved equilibrium stress growth law, based on the small strain version developed for the $9 \mathrm{Cr}-1 \mathrm{Mo}$ steel, now allows us to incorporate any of these choices of kinematic stress growth laws, as well as rate hyperelasticity if necessary; this was not possible with the previous version. We have implemented, for example, a corotational kinematic stress rule, based on an Armstrong-Frederick format, and modeled shear and second order axial stresses which arise during torsion of an axially constrained thin walled tube. The results show good qualitative and quantitative agreement with experimental data of 316 Stainless Steel and 1100 Aluminum. A brief representation of results is shown by Majors and Krempl in a paper to be presented at the forthcoming Fourth International Symposium on Plasticity and Its Current Applications.

\section{Experimental Studies}

The aim of the experimental research is to measure the mechanical response and the changes in the potential drop during high temperature biaxial low-cycle fatigue of Type 304 Stainless Steel, an alloy for primary structures in high temperature nuclear reactors. Reversing direct current is used to measure the voltage drop between probes spot welded to the tubular specimen gauge section while the specimen is being subjected to biaxial (axial-torsion) loadings in the MTS servohydraulic computer-controlled testing machine. The gauge section wall thickness was reduced by $10 \%$ to promote crack formation there. The specimen is cycled to failure in tests lasting up to 18 hours. Temperature in the entire gauge length does not vary by more than $3^{\circ} \mathrm{C}$ from the desired temperature for the entire test. Electrical interference from the induction heater is overcome by using an analog filter and a long voltmeter integration time. Several megabytes of millivolt-range data are gathered in a single test.

Seven low cycle fatigue tests (two axial, four in-phase proportional axial-torsion, and one torsional test) with a sinusoidal command signal input were completed. In all tests, the potential drop increased uniformly at all circumferential positions on the specimen. Since the voltage data is periodic from axial cyclic straining, a Fourier interpolation technique was used to identify the per-cycle potential drop maxima. A one-dimensional piecewise cubic spline and a two-dimensional tensor product spline were used to interpolate these maxima with respect to time and spatial position. The potential drop is normalized with respect to the starting value. The increment of normalized potential drop at any position is calculated with respect to the lowest normalized potential drop at any time. The resulting incremental normalized potential drop is plotted as a surface to make spatial and time variations apparent.

The results are consistent. Before a crack forms and grows to detectable size, the potential drop increases uniformly around the specimen, due to non-localized resistance changes from oxidation and other causes. When a crack forms, a small hill appears in the plot and grows and spreads as the crack grows. 
The experimental results are compared with the potential drop predicted by electrostatic theory. Several analytic solutions to Laplace's equation predict potential drops that are in close agreement with theory. A paper on this subject was presented at the Eleventh Symposium on Energy Engineering Sciences.

\section{Further Work}

The principal investigator has received a Humboldt Senior Scientist Award. He will be on sabbatical starting September 1993 and will continue to direct the research and supervise the graduate students via electronic mail and return visits. Collaborations with the Technische Hochshule Darmstadt, Professor Kollmann, on finite deformation and with Universität Erlangen, Professor Kuhn, on damage mechanics are planned. Mr. Majors is expected to be graduating this fall. Mr. Fitzgerald anticipates defending his thesis in spring of 1994. A new student will have to be hired and is expected to start in the fall. We expect to purchase new test material which will be investigated by the new student.

We are continuing numerical tests of other specializations of the finite deformation model. Particular attention is being paid to second order stresses and displacements resulting from constrained and free end torsion of thin walled tubes. We are also summarizing our kinematic analysis so as to show the relations to other models in finite plasticity, including single crystal analyses, with the goal of allowing easy specialization of VBO to various materials as data becomes available.

The experimental program will continue with more axial testing and new $90^{\circ} \mathrm{deg}$ out-of-phase axial-torsion tests. Additional computer simulations of the electrostatic field will be performed using other analytic solutions to Laplace's equation and finite difference approximations, to obtain closer simulation to observed phenomena. The resulting crack growth data will be incorporated into a crack growth law, which will be compared to the results of others. 


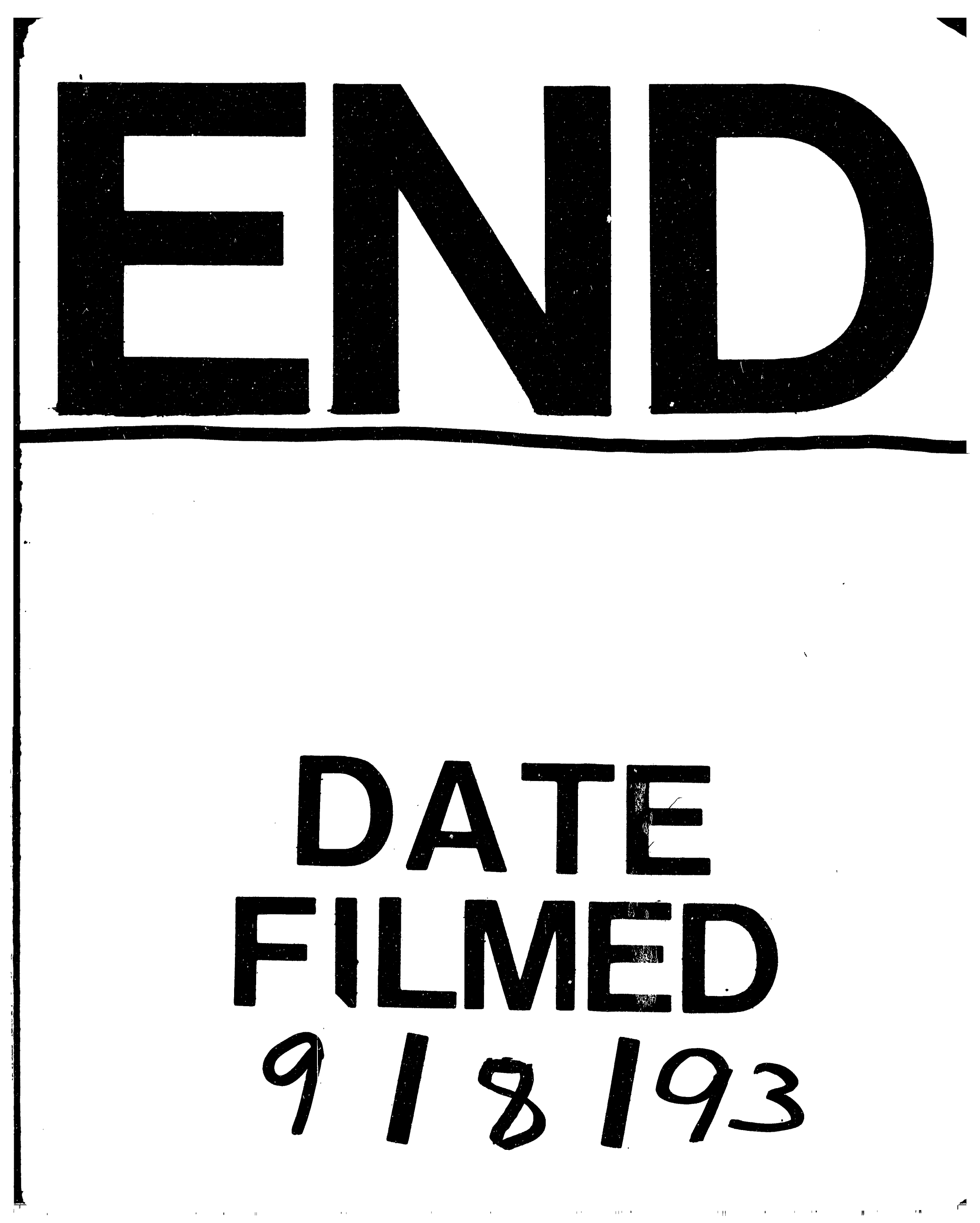




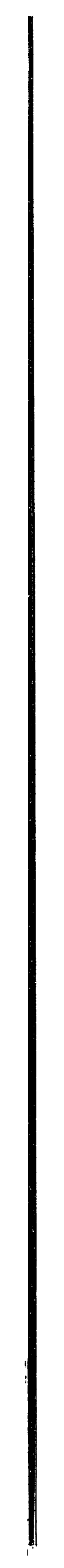

